01

\title{
Усиление интенсивности комбинационного рассеяния за счет фактора внутреннего поля
}

\author{
() Д.В. Петров ${ }^{1,2}$, И.И. Матросов ${ }^{1}$ \\ ${ }^{1}$ Институт мониторинга климатических и экологических систем Сибирского отделения РАН, \\ 634055 Томск, Россия \\ 2 Томский государственный университет, \\ 634050 Томск, Россия \\ e-mail: dpetrov@imces.ru
}

Поступила в редакцию 03.11.2020 г.

В окончательной редакции 03.11.2020 г.

Принята к публикации 05.02.2021 г.

Рассмотрено усиление интенсивности сигналов комбинационного рассеяния (КР) компонентов газовых сред за счет фактора внутреннего поля, создаваемого молекулярным окружением. Исследована интенсивность фундаментальной колебательно-вращательной полосы КР азота в смесях с аргоном и метаном при давлениях до $50 \mathrm{~atm}$. Установлено, что интенсивность сигналов КР фиксированной концентрации азота в данных смесях варьирует в пределах 4\% в зависимости от давления и окружения. Представлена модель, описывающая данный эффект. Полученные данные позволят повысить точность газоанализа, основанного на спектроскопии КР.

Ключевые слова: комбинационное рассеяние света, усиление интенсивности сигналов, внутреннее поле, метан, азот.

DOI: $10.21883 /$ OS.2021.05.50880.272-20

\section{Введение}

За последние 10-20 лет произошло значительное улучшение чувствительности газоанализаторов, основанных на спектроскопии комбинационного рассеяния (КР) света. На сегодняшний день минимально определяемая концентрация с помощью таких устройств составляет менее 1 ppm [1-3], что открывает возможности для их применения в области контроля таких многокомпонентных газовых сред как природный газ [4-7], выдыхаемый воздух [8-10], атмосферный воздух $[3,11]$ и т. п. Наиболее вероятно, что в ближайшие годы чувствительность КРгазоанализаторов будет еще выше за счет улучшения характеристик фотодетекторов, а также развития методов увеличения интенсивности информативных сигналов $[2,6,10,12-17]$. Однако для извлечения наиболее достоверных значений концентраций из спектров КР необходим учет ряда особенностей, которые ранее игнорировались ввиду низких значений сигнал/шум. Одной из них является взаимосвязь между интенсивностью сигналов КР и концентрацией молекул. Вопреки распространенному мнению о линейной зависимости между данными величинами экспериментальные результаты [18-20] свидетельствуют об отклонении от линейной связи. Это объясняется тем, что в зависимости от плотности среды в месте нахождения рассматриваемой молекулы изменяется электрическое поле, что приводит к изменению интенсивности сигналов КР [21]. Данный эффект носит название фактор внутреннего поля и, согласно [22], связан функциональной зависимостью с показателем преломления среды. Наиболее наглядно этот фактор проявляется на примере увеличения эффективных сечений рассеяния (произведение сечения рассеяния на фактор внутреннего поля) молекул в жидкости по сравнению с газом [18]. Согласно работам [19,20], данный эффект также проявляется при изменении давления газовой среды (без изменения агрегатного состояния), хотя в значительно меньшей степени. На основании совокупности результатов логичным является предположение о том, что изменение молекулярного окружения также может приводить к вариациям интенсивностей сигналов КР, приходящихся на единицу концентрации молекул. В настоящее время данный вопрос достаточно актуален, поскольку на практике в случае определения концентраций с помощью метода разложения спектра КР многокомпонентной смеси на спектры отдельных компонентов [7] эффект изменения интенсивностей может приводить к увеличению погрешности измерений, так как опорные спектры и спектры смеси могут быть получены в различных условиях (давление и/или окружение). В этой связи целью данной работы являлось получение и верификация модели, описывающей изменение интенсивностей сигналов КР в газовых смесях.

\section{Теория}

В плотных газовых средах интенсивность сигналов КР отдельной колебательно-вращательной полосы $v_{j}$ спектра $I_{R}$ из объема $V$ в телесный угол $\Delta \Omega$ для молекул 
одного сорта может быть представлена в виде

$$
I_{R}=I_{L} V \sigma_{j} \Delta \Omega \frac{P}{k T Z(P, T)} L(P) S(P),
$$

где $I_{L}$ - интенсивность возбуждающего лазерного излучения, $\sigma_{j}$ - дифференциальное сечение рассеяния (для фиксированного угла наблюдения рассеянного излучения к направлениям поляризации и распространения возбуждающего излучения), $k-$ постоянная Больцмана, $T$ - температура, $P$ - давление, $Z(P, T)$ - коэффициент сжимаемости газа, $L(P)$ - фактор внутреннего поля, $S(P)$ - коэффициент, описывающий изменение угла сбора при давлении $P$ по сравнению с углом сбора при $1 \mathrm{~atm}$ (инструментальный фактор). Далее для упрощения формул обозначения зависимости от температуры и давления для величин $Z, L$ и $S$ будут опущены.

Рассмотрим фактор внутреннего поля смеси $L_{\text {mix }}$. В соответствии с [22]

$$
L_{\operatorname{mix}} \approx\left[\frac{\left(n_{\mathrm{mix}}\right)^{2}+2}{3}\right]^{4},
$$

где $n_{\text {mix }}-$ показатель преломления смеси на частоте лазерного излучения.

Используя молекулярную рефракцию газа, данный фактор может быть представлен [20] в виде

$$
L_{\mathrm{mix}} \approx\left[1-\left(\frac{P_{\mathrm{mix}} A_{\mathrm{mix}}^{R}}{R T Z_{\mathrm{mix}}}+\frac{P_{\mathrm{mix}}^{2} B_{\mathrm{mix}}^{R}}{R^{2} T^{2} Z_{\mathrm{mix}}^{2}}\right)\right]^{-4},
$$

где $A_{\mathrm{mix}}^{R}$ и $B_{\mathrm{mix}}^{R}-$ первый и второй вириальные коэффициенты рефракции смеси, $P_{\operatorname{mix}}$ и $Z_{\operatorname{mix}}$ - давление и коэффициент сжимаемости смеси соответственно. Коэффициенты $A_{\mathrm{mix}}^{R}$ и $B_{\mathrm{mix}}^{R}$ связаны с вириальными коэффициентами отдельных компонентов смеси соотношениями

$$
\begin{gathered}
A_{\mathrm{mix}}^{R}=\sum_{i=1}^{n} x_{i} A_{i}^{R}, \\
B_{\mathrm{mix}}^{R}=\sum_{i=1}^{n} \sum_{j=1}^{n} x_{i} x_{j} B_{i j}^{R},
\end{gathered}
$$

где $n$ - количество компонентов смеси, $x_{i}$ - доля молекул $i$-го сорта в смеси. Отметим, что $B_{i i}^{R} \equiv B_{i}^{R}$. Если вириальные коэффициенты $B_{i j}^{R}$ неизвестны, то их можно оценить [23] как

$$
B_{i j}^{R}=\sqrt{B_{i}^{R} B_{j}^{R}} .
$$

Коэффициент сжимаемости смеси $Z_{\text {mix }}$ может быть выражен через коэффициенты сжимаемости ее отдельных компонентов $Z_{i}$ и состав смеси с помощью следующего уравнения:

$$
Z_{\mathrm{mix}}=1-\left\{\sum_{i=1}^{n} x_{i} \sqrt{\left[1-Z_{i}\right]}\right\}^{2}
$$

В свою очередь, $Z_{i}$ могут быть найдены посредством решения уравнения

$$
Z_{i}^{3}-Z_{i}^{2}-\frac{P B(T)}{R T} Z_{i}-\frac{P^{2} C(T)}{R^{2} T^{2}}=0,
$$

где $B(T)$ и $C(T)$ - второй и третий вириальные коэффициенты сжимаемости.

\section{Эксперимент}

Согласно (1) и (2), интенсивность сигналов КР компонента $i$ может быть увеличена за счет фактора внутреннего поля, которое создается окружающими молекулами. Для проверки этого заключения мы провели исследование двух бинарных смесей. Первая состояла из азота (в качестве основного газа) и аргона (в качестве буферного газа). Азот имеет только одну фундаментальную полосу и, следовательно, в его спектре исключены перераспределения интенсивностей при различных условиях (давление или окружение). Поскольку аргон является атомарным газом, то он не имеет спектра КР. Таким образом, изменение интенсивности сигналов азота фиксированной концентрации в смеси с аргоном может быть обусловлено только инструментальными особенностями либо фактором внутреннего поля. Вторая смесь состояла из азота (в качестве основного газа) и метана (в качестве буферного газа). По сравнению с аргоном метан имеет другую сжимаемость и рефракцию (табл. 1), следовательно, для данных газов фактор внутреннего поля при эквивалентных давлениях будет различаться.

Для регистрации спектров КР была использована экспериментальная установка, описанная в [7,20]. В данной установке для сбора рассеянного света вместо пары линзовых объективов с относительным отверстием $1: 1.8$ и фокусным расстоянием $f=50 \mathrm{~mm}$ были использовали два объектива с относительным отверстием $1: 4$ и фокусным расстоянием $f=105 \mathrm{~mm}$ для минимизации инструментального фактора при увеличении давления. На первом этапе был зарегистрирован спектр КР чистого азота при давлении $1 \mathrm{~atm}$. После этого были получены спектры смесей $\mathrm{N}_{2}(1 \mathrm{~atm})+\mathrm{Ar}(P)$ и $\mathrm{N}_{2}(1 \mathrm{~atm})+\mathrm{CH}_{4}(P)$, где $P$ варьировалось от 4 до $49 \mathrm{~atm}$ с шагом $5 \mathrm{~atm}$. Для повышения достоверности экспериментальных результатов данный цикл измерений был проведен пять раз. Чистота используемых газов была > 99.99\%. Смеси подготавливались в предварительной смесительной камере $\left(V=2000 \mathrm{~cm}^{3}\right)$ и после этого наполнялась кювета $\left(V=10 \mathrm{~cm}^{3}\right)$. Давление азота при приготовлении смесей контролировалось манометром с погрешностью $0.02 \%$. На основании этого можно утверждать, что концентрация анализируемых молекул азота во всех смесях была стабильна с данной точностью. Давление смеси контролировалось манометром с погрешностью $0.05 \%$. Мощность лазера контролировалось с помощью фотодиода с погрешностью $<0.1 \%$. Время экспозиции 
Таблица 1. Значения вириальных коэффициентов для азота, аргона и метана при $T=300 \mathrm{~K}$

\begin{tabular}{c|c|c|l|l}
\hline Газ & $B, \mathrm{~cm}^{3} / \mathrm{mol}$ & $C, \mathrm{~cm}^{6} / \mathrm{mol}^{2}$ & $A^{R}, \mathrm{~cm}^{3} / \mathrm{mol}$ & $B^{R}, \mathrm{~cm}^{6} / \mathrm{mol}^{2}$ \\
\hline $\mathrm{N}_{2}$ & $-4.5[24]$ & $1500[24]$ & $4.4464[25]$ & $0.89[25]$ \\
$\mathrm{Ar}$ & $-15[24]$ & $1080[24]$ & $4.197[25]$ & $2[25]$ \\
$\mathrm{CH}_{4}$ & $-43[24]$ & $2400[24]$ & $6.576[25]$ & $6.08[25]$
\end{tabular}

каждого спектра составляло 500 s. Температуры воздуха в лаборатории и газовой кюветы были $300 \pm 1 \mathrm{~K}$.

\section{Результаты и обсуждение}

Особенностью используемого КР-спектрометра является пренебрежимо малая фоновая засветка, источником которой зачастую является рассеяние от стенок кюветы или оптических элементов. В этой связи перед процедурой определения интенсивностей никакой предварительной обработки спектров $\mathrm{N}_{2}+\mathrm{Ar}$ не проводилось. В свою очередь, в спектрах $\mathrm{N}_{2}+\mathrm{CH}_{4}$ в области полосы азота наблюдался фон, обусловленный рассеянием от интенсивных линий пентады метана $2 v_{4}: v_{2}+v_{4}: v_{1}: v_{3}: 2 v_{2}$. Для его учета из каждого спектра $\mathrm{N}_{2}(1 \mathrm{~atm})+\mathrm{CH}_{4}(P)$ был вычтен спектр чистого $\mathrm{CH}_{4}$, зарегистрированный при давлении $P$. В результате данной операции фактически был получен спектр азота, спектральные характеристики которого обусловлены окружением метана.

На рис. 1 показаны зарегистрированные спектры КР азота при различном окружении. Можно видеть, что его интенсивность в средах с аргоном и метаном несколько увеличилась. Для получения количественных оценок из всех зарегистрированных спектров азота была определена интегральная интенсивность в диапазоне $2200-2500 \mathrm{~cm}^{-1}$. В спектрах азота наблюдались сдвиг (до $0.45 \mathrm{~cm}^{-1}$ ) и уширение $Q$-ветви (до $0.15 \mathrm{~cm}^{-1}$ ), вызванные увеличением давления. Отметим, что с учетом сравнительно широкого диапазона, в котором проводилось интегрирование интенсивностей, данные спектральные изменения не оказывали влияния на полученные значения.

Поскольку при изменении давления в кювете изменяется показатель преломления, то это приводит к изменению эффективного фокусного расстояния объектива, с помощью которого собирается рассеянное излучение. В результате изменяется телесный угол сбора $\Delta \Omega$, входящий в соотношение (1), что приводит к изменению зарегистрированной интенсивности. Для оценки данного инструментального фактора было сделано следующее. Поскольку данная величина зависит от показателя преломления, то мы рассчитали увеличение эффективного фокусного расстояния объектива, предназначенного для сбора рассеянного света, при различных $n_{\text {mix }}$. Требуемые показатели преломления для этого рассчитывались с помощью уравнения (2), $L_{\mathrm{mix}}$ в котором вычислялось с помощью уравнений (3)-(8). Максимальное увеличение фокусного расстояния составило $0.22 \mathrm{~mm}$ для смеси $\mathrm{N}_{2}(1 \mathrm{~atm})+\mathrm{CH}_{4}(49 \mathrm{~atm})$. После этого мы зарегистрировали набор спектров КР чистого азота при фиксированном давлении $1 \mathrm{~atm}$ посредством установки данного объектива на различном расстоянии в диапазоне $0-0.22 \mathrm{~mm}$ от оптической оси лазерного излучения, проходящего внутри кюветы. Настройка экспериментальной установки и все параметры регистрации спектров были неизменными. С помощью аппроксимации полученных данных мы получили зависимость изменения интенсивности от показателя преломления газа внутри кюветы. Принимая во внимание рассчитанные значения $n_{\text {mix }}$ (табл. 2), были получены значения инструментального фактора для каждой смеси.

На рис. 2 представлены полученные усредненные экспериментальные значения интегральной интенсивности излучения азота для различного давления и окружения, нормированные на интегральную интенсивность при $1 \mathrm{~atm}$. Доверительные интервалы представляют собой среднеквадратическое отклонение, полученное из 5 измерений. Можно видеть, что в обеих смесях интенсивность увеличивалась с давлением буферного газа, при этом в среде метана это увеличение было больше. Коэффициент увеличения достигает $4 \%$ в смеси $\mathrm{N}_{2}(1 \mathrm{~atm})+\mathrm{CH}_{4}(49 \mathrm{~atm})$. Отметим, что с учетом доверительных интервалов экспериментальные данные достаточно близки к теоретическим, что говорит о правомерности представленной выше модели.

Рассмотрим влияние данного эффекта на точность КР-газоанализа. Наиболее эффективным методом вычисления концентраций в многокомпонентных смесях (например, природный газ), где наблюдается значительное перекрытие линий спектров различных компонентов, является разложение спектра смеси на спектры отдельных компонентов [7]. Однако для реализации анализа природного газа (ПГ) с помощью данного метода in situ необходима специфическая подготовка базисных спектров. Это обусловлено тем, что для минимизации погрешностей все базисные спектры должны иметь спектральные характеристики (полуширины и сдвиг) эквивалентные тем условиям, при которых получен спектр образца ПГ [26,27]. В свою очередь, давление ПГ в магистральных трубопроводах может достигать $100 \mathrm{~atm}$, однако спектры чистых углеводородов $\mathrm{C} 2+$ не могут быть получены в газообразном состоянии при таком давлении при комнатной температуре. Например, при $T=300 \mathrm{~K}$ максимальное давление пропана составляет примерно $6 \mathrm{~atm}$, бутанов $\sim 2 \mathrm{~atm}$, пентанов $\sim 0.5 \mathrm{~atm}$ и т.д. В связи с этим при подготовке данных базисных спектров вместо чистых углеводородов могут быть использованы бинарные смеси с буферным газом. В качестве последнего целесообразно использовать атомарный газ (например, аргон) по причине отсутствия спектра КР. За счет вариации давления буферного газа в данной смеси возможно получить спектр любого углеводорода с полуширинами и сдвигом линий, близкими к тем, 
Таблица 2. Значения показателя преломления, фактора сжимаемости и фактора внутреннего поля для смесей $\mathrm{N}_{2}(1 \mathrm{~atm})+\mathrm{CH}_{4}(P)$ и $\mathrm{N}_{2}(1 \mathrm{~atm})+\operatorname{Ar}(P)$

\begin{tabular}{r|c|c|c|c|c|c}
\hline \multirow{2}{*}{$P$, atm } & \multicolumn{3}{|c|}{$\mathrm{N}_{2}(1 \mathrm{~atm})+\mathrm{CH}_{4}(P)$} & \multicolumn{3}{|c}{$\mathrm{N}_{2}(1 \mathrm{~atm})+\operatorname{Ar}(P)$} \\
\cline { 2 - 7 } & $n_{\text {mix }}$ & $Z_{\text {mix }}$ & $L_{\text {mix }}$ & $n_{\text {mix }}$ & $Z_{\text {mix }}$ & $L_{\text {mix }}$ \\
\hline 0 & 1.00027 & 0.9998 & 1.0007 & 1.00027 & 0.9998 & 1.0007 \\
4 & 1.00188 & 0.9952 & 1.0050 & 1.00136 & 0.9982 & 1.0035 \\
9 & 1.00393 & 0.9870 & 1.0106 & 1.00272 & 0.9955 & 1.0069 \\
14 & 1.00602 & 0.9786 & 1.0162 & 1.00408 & 0.9927 & 1.0104 \\
19 & 1.00814 & 0.9702 & 1.0219 & 1.00544 & 0.9899 & 1.0139 \\
24 & 1.01031 & 0.9618 & 1.0279 & 1.0068 & 0.9872 & 1.0175 \\
29 & 1.01251 & 0.9535 & 1.034 & 1.00817 & 0.9846 & 1.0211 \\
34 & 1.01476 & 0.9453 & 1.0402 & 1.00953 & 0.9820 & 1.0247 \\
39 & 1.01704 & 0.9372 & 1.0466 & 1.0109 & 0.9795 & 1.0284 \\
44 & 1.01936 & 0.9293 & 1.0532 & 1.01226 & 0.9771 & 1.0321 \\
49 & 1.02172 & 0.9215 & 1.0599 & 1.01362 & 0.9748 & 1.0358
\end{tabular}
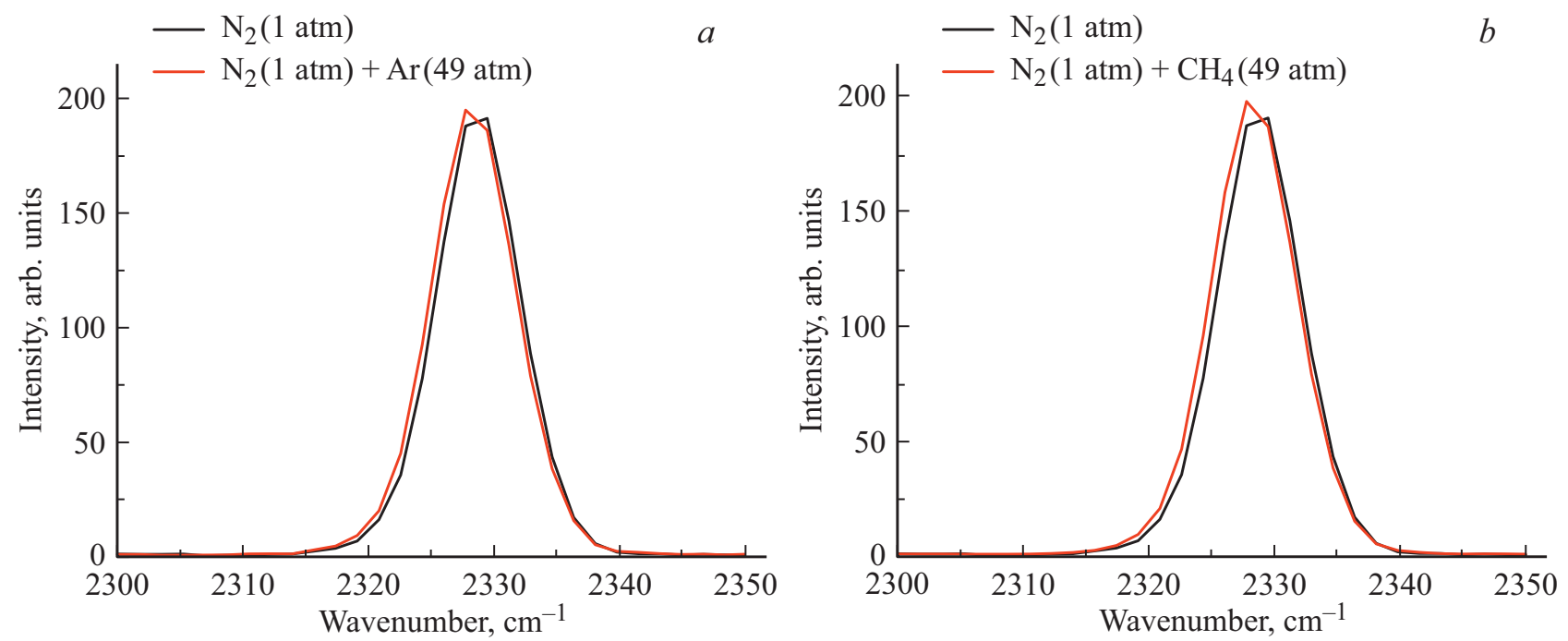

Рис. 1. Спектры КР азота в среде аргона $(a)$ и в среде метана $(b)$ по сравнению с чистым азотом.
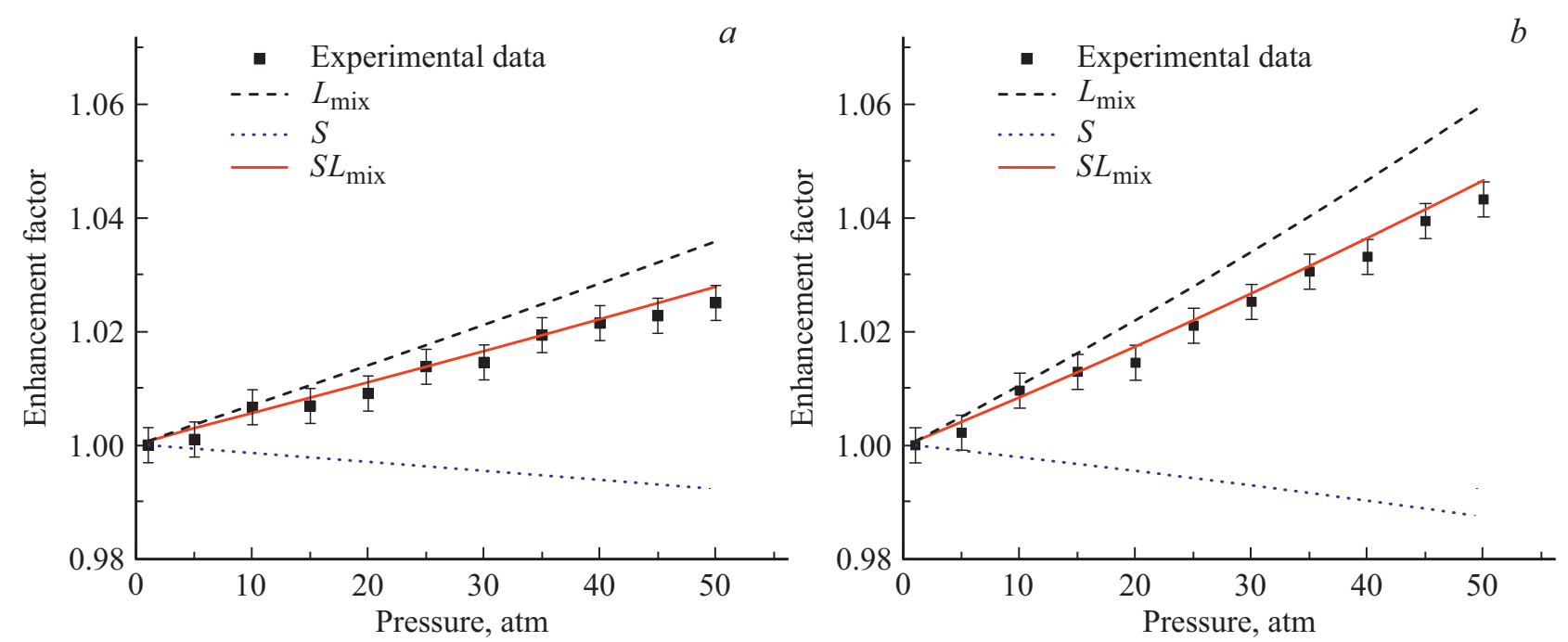

Рис. 2. Значения фактора внутреннего поля $L_{\mathrm{mix}}$, инструментального фактора $S$, их произведение $S L_{\text {mix }}$, а также экспериментальные значения коэффициента увеличения интегральной интенсивности азота в виде функции давления смеси $\mathrm{N}_{2}+\mathrm{Ar}_{(a)} \mathrm{N}_{2}+\mathrm{CH}_{4}(b)$. 
которые наблюдаются в образце ПГ. Принимая во внимание эффект внутреннего поля, интенсивность сигналов КР в данных смесях будет увеличена по сравнению со спектрами чистых компонентов. Согласно рис. 2, при использовании аргона при $P=50 \mathrm{~atm}$ это увеличение составит примерно 2\%. Это приведет к тому, что погрешность определения концентрации данного углеводорода возрастет на эту величину. Поскольку целью многих разработчиков КР-газоанализаторов является замена газовых хроматографов, то увеличение погрешности на $2 \%$ является значительным, поскольку метод газовой хроматографии обеспечивает погрешность в пределах $6 \%$ [28]. В связи с этим для повышения точности измерений эффект внутреннего поля должен быть учтен.

\section{Заключение}

Представленные данные показали, что за счет внутреннего поля, создаваемого окружающими молекулами, возможно увеличение интенсивностей сигналов КР анализируемого газа. С одной стороны, на практике это может быть реализовано, например, посредством добавления значительного объема любого атомарного газа (ввиду отсутствия спектров КР). При больших давлениях это может привести к увеличению интенсивности на десятки процентов. С другой стороны, описанные эффекты должны быть учтены в процедуре определения концентраций из спектров КР для повышения точности измерений.

\section{Финансирование работы}

Работа выполнена при поддержке Российского фонда фундаментальных исследований (грант № 19-42-700006).

\section{Конфликт интересов}

Авторы заявляют, что у них нет конфликта интересов.

\section{Список литературы}

[1] Petrov D.V., Matrosov I.I., Tikhomirov A.A. // J. Appl. Spectrosc. 2015. V. 82. N 1. P. 120. doi 10.1007/s10812-015-0073-4

[2] Gomez Velez J., Muller A. // Opt. Lett. 2020. V. 45. N 1. P. 133. doi 10.1364/OL.45.000133

[3] Hanf S., Keiner R., Yan D., Popp J., Frosch T. // Anal. Chem. 2014. V. 86. N 11. P. 5278. doi 10.1021/ac404162w

[4] Sharma R., Poonacha S., Bekal A., Vartak S., Weling A., Tilak V., Mitra C. // Opt. Eng. 2016. V. 55. N 10. P. 104103. doi 10.1117/1.OE.55.10.104103

[5] Sieburg A., Knebl A., Jacob J.M., Frosch T. // Anal. Bioanal. Chem. 2019. V. 411. N 28. P. 7399. doi $10.1007 / \mathrm{s} 00216-019-02145-\mathrm{x}$

[6] Hippler M. // Anal. Chem. 2015. V. 87. N 15. P. 7803. doi 10.1021/acs.analchem.5b01462
[7] Petrov D.V., Matrosov I.I. // Appl. Spectrosc. 2016. V. 70. N 10. P. 1770 . doi $10.1177 / 0003702816644611$

[8] Chow K.K., Short M., Lam S., Mc Williams A., Zeng H. // Med. Phys. 2014. V. 41. N 9. P. 092701. doi 10.1118/1.4892381

[9] Bögózi T., Popp J., Frosch T. // Bioanalysis. 2015. V. 7. N 3. P. 281. doi 10.4155/bio.14.299

[10] Petrov D.V. // Appl. Opt. 2016. V. 55. N 33. P. 9521. doi 10.1364/AO.55.009521

[11] Petrov D.V., Matrosov I.I., Zaripov A.R. // J. Mol. Spectrosc. 2018. V. 348. P. 137-141. doi 10.1016/j.jms.2018.01.001

[12] Petrov D.V., Zaripov A.R., Toropov N.A. // Opt. Lett. 2017. V. 42. N 22. P. 4728. doi 10.1364/OL.42.004728

[13] Schlüter S., Krischke F., Popovska-Leipertz N., Seeger T., Breuer G., Jeleazcov C., Schüttler J., Leipertz A. // J. Raman Spectrosc. 2015. V. 46. N 8. P. 708. doi 10.1002/jrs.4711

[14] Knebl A., Yan D., Popp J., Frosch T. // Trends Anal. Chem. 2018. V. 103. P. 230. doi 10.1016/j.trac.2017.12.001

[15] Yu A., Zuo D., Li B., Gao J., Wang X. // Appl. Opt. 2016. V. 55. N 13. P. 3650. doi 10.1364/AO.55.003650

[16] Buric M.P., Chen K.P., Falk J., Woodruff S.D. // Appl. Opt. 2009. V. 48. N 22. P. 4424. doi 10.1364/AO.48.004424

[17] Wang P., Chen W., Wan F., Wang J., Hu J. // Opt. Express. 2019. V. 27. N 23. P. 33312. doi 10.1364/OE.27.033312

[18] Nestor J.R., Lippincott E.R. // J. Raman Spectrosc. 1973. V. 1. N 3. P. 305. doi 10.1002/jrs.1250010309

[19] Oksengorn B., Fabre D. // Appl. Spectrosc. 1992. V. 46. N 3. P. 468. doi 10.1366/0003702924125348

[20] Petrov D.V., Matrosov I.I. // J. Raman Spectrosc. 2017. V. 48. N 3. P. 474. doi $10.1002 /$ jrs. 5062

[21] Schrötter H.W., Klöckner H.W. Raman Scattering Cross Sections in Gases and Liquids // Raman spectroscopy of gases and liquids / Ed. by Weber A. Berlin: Springer-Verlag, 1979. $320 \mathrm{p}$.

[22] Eckhardt G., Wagner W.G. // J. Mol. Spectrosc. 1966. V. 19. N 1-4. P. 407. doi 10.1016/0022-2852(66)90262-1

[23] Achterman H.J., Bose T.K., Jaeschke M., St-Arnaud J.M. // Int. J. Thermophys. 1986. V. 7. N 2. P. 357. doi 10.1007/BF00500161

[24] Dymond J.H., Marsh K.N., Wilhoit R.C., Wong K.C. Virial coefficients of pure gases and mixtures // Virial coefficients of pure gases / Ed. by Frenke M., Marsh K.N. Berlin: Springer-Verlag, 2003. $327 \mathrm{p}$.

[25] Achtermann H.J., Magnus G., Bose T.K. // J. Chem. Phys. 1991. V. 94. N 8. P. 5669. doi $10.1063 / 1.460478$

[26] Petrov D.V., Matrosov I.I., Zaripov A.R., Maznoy A.S. // Spectrochim. Acta A. 2019. V. 215. P. 363. doi 10.1016/J.SAA.2019.03.006

[27] Petrov D. V., Matrosov I.I., Zaripov A.R., Maznoy A.S. // Appl. Spectrosc. 2020. V. 74. N 8. P. 948. doi $10.1177 / 0003702820917222$

[28] ГОСТ 31371.7-2008. Газ природный. Определение состава методом газовой хроматографии с оценкой неопределенности. Часть 7. Методика выполнения измерений молярной доли компонентов. 